\title{
Co-transduction of ribosomal protein L23 enhances the therapeutic efficacy of adenoviral-mediated p53 gene transfer in human gastric cancer
}

\author{
YA-FEI ZHANG ${ }^{1,2^{*}}$, BI-CHENG ZHANG ${ }^{1 *}$, AN-RAN ZHANG ${ }^{2}$, TING-TING WU ${ }^{1}$, JIAN LIU ${ }^{1}$, \\ LI-FANG YU ${ }^{1}$, WEI-XING WANG ${ }^{1}$, JIAN-FEI GAO ${ }^{1}$, DIAN-CHUN FANG ${ }^{2}$ and ZHI-GUO RAO ${ }^{1}$ \\ ${ }^{1}$ Department of Oncology, Wuhan General Hospital of Guangzhou Command, People's Liberation Army, \\ Wuhan, Hubei 430070; ${ }^{2}$ Department of Gastroenterology, Southwest Hospital, \\ Third Military Medical University, Chongqing 400038, P.R. China
}

Received June 14, 2013; Accepted July 17, 2013

DOI: 10.3892/or.2013.2663

\begin{abstract}
Induction of murine double minute 2 (MDM2) expression is thought to be a determinant of resistance to p53 gene therapy for cancer. Previous studies have revealed that ribosomal protein L23 (RPL23) inhibits MDM2-mediated p53 degradation through direct binding to MDM2. In addition, ectopically expressed RPL23 was reported to interact with MDM2 in both the nucleus and cytoplasm, by which RPL23 indirectly inhibited MDM2-p53 binding. Based on the known molecular properties of the RPL23 protein, it was speculated that co-transduction of RPL23 may protect wild-type p53 protein from MDM2-mediated inactivation and, thus, improve the effect of delivering therapeutic exogenous p53. To test this hypothesis, we constructed a bicistronic adenoviral vector expressing both the RPL23 and p53 genes (Ad-RPL23/p53) and compared its tumor-suppressor activity in human gastric cancer with that of a single gene vector for p53 (Ad-p53). In the in vivo and in vitro experiments, we observed that treatment with Ad-RPL23/p53 resulted in a stronger antitumor response compared to that obtained using Ad-p53. Moreover, the antitumor response of the bicistronic adenovirus was obtained not only in MGC803 cells (endogenous mutant p53) but also in MKN45 cells (endogenous wild-type p53) which
\end{abstract}

Correspondence to: Dr Zhi-Guo Rao, Department of Oncology, Wuhan General Hospital of Guangzhou Command, People's Liberation Army, Wuhan, Hubei 430070, P.R. China

E-mail: raozhiguo2012@163.com

Dr Dian-Chun Fang, Department of Gastroenterology, Southwest Hospital, Third Military Medical University, Chongqing 400038, P.R. China

E-mail: fangdianchun@hotmail.com

*Contributed equally

Key words: ribosomal protein L23, p53, murine double minute 2, gene therapy, gastric cancer were initially resistant to p53 gene transfer, indicating that co-transduction of RPL23 also expanded the utility of p53 gene therapy. Furthermore, in an orthotopic nude mouse model of human gastric cancer, we found that the survival benefit was greater after Ad-RPL23/p53 treatment than after Ad-p53. Taken together, the data presented here demonstrate that co-transduction of RPL23 enhances the therapeutic efficacy of adenoviral-mediated p53 gene transfer in models of human gastric cancer and support the use of this strategy for cancer treatment.

\section{Introduction}

During the past 20 years, various therapeutic approaches based on the p53 pathway have been developed $(1,2)$. Among them, therapeutic application of adenoviral-mediated p53 gene transfer has been most widely investigated (1). Although efficacy without non-specific toxicity is noted in both tissue culture and animal models, responses of adenoviral-mediated p53 gene transfer in early human trials have not been spectacular. This is due, in part, to the observation that a substantial fraction of tumor cells, particularly tumor cells exhibiting wild-type (wt) p53, are resistant to p53 gene transfer $(3,4)$.

A large body of evidence has established murine double minute 2 (MDM2) (also termed HDM2 for its human equivalent) as a crucial negative regulator of p53 and the major suppressor of p53 function in tumors with wt p53. MDM2 binds p53 and functions as a ubiquitin E3 ligase to promote p53 ubiquitination and degradation by proteasomes (5-7). As the MDM2 gene itself is a downstream target of p53, induction of MDM2 establishes a negative feedback loop to limit the action of p53, in which p53 itself initiates its own destruction $(8,9)$. Therefore, it has been suggested that MDM2 may be potentially responsible for the resistance of tumors to p53 gene transfer, in which, overexpression of MDM2 or exogenous p53-induced overexpression of MDM2, leads to rapid degradation of the p53 protein (10-13).

Ribosomal protein L23 (RPL23) was reported to have the capability to inhibit MDM2-mediated p53 ubiquitination and degradation through direct binding to MDM2 $(14,15)$. In 
addition, ectopically expressed RPL23 was found to interact with MDM2 in both the cytoplasm and the nucleus, which indicates that MDM2 was retained in the cytoplasm and the nucleus as a complex, and this complex formation represents one more mechanism by which RPL23 indirectly inhibits MDM2-p53 binding (14). A previous study by our group showed that, through inhibition of MDM2-p53 interaction, adenoviral-mediated expression of RPL23 displayed modest tumor-suppressor activity in vitro and in vivo in human gastric cancer MKN45 cells with endogenous wild-type p53, which were initially resistant to the p53 gene transfer $(12,16,17)$. In the present study, using a RPL23/p53 bicistronic adenovirus, we aimed to determine whether co-transduction of the RPL23 gene enhances the therapeutic efficacy of adenoviral-mediated p53 gene transfer through protecting exogenous p53 protein from MDM2-mediated inactivation in human gastric cancer cells.

\section{Materials and methods}

Cell lines and reagents. Two human gastric cancer cell lines, MKN45 (endogenous wt p53 gene) and MGC803 (endogenous mutant p53 gene), were used in this study $(18,19)$. Both cells lines were maintained in RPMI-1640 supplemented with $10 \%$ fetal calf serum (FCS) (Invitrogen Life Technologies) at $37^{\circ} \mathrm{C}$ in a humidified atmosphere containing $5 \% \mathrm{CO}_{2}$. Mouse monoclonal antibodies specific for p53 (ab-4, specific for wild-type p53), MDM2, p21 and PUMA were obtained from Santa Cruz Biotechnology, Inc. (Santa Cruz, CA, USA), and mouse monoclonal $\beta$-actin antibody was obtained from Sigma-Aldrich Chemical Co. (St. Louis, MO, USA). All reagents were used in different concentrations as indicated.

Construction of recombinant adenovirus. A replication defective adenoviral vector, deleted in E1A, E1B and E3, was constructed in which the viral E1A and E1B genes were replaced with a bicistronic cassette encoding RPL23 and p53 under the control of the CMV promoter (Ad-RPL23/p53), using the AdEasy vector system (Wuhan Genesil Biotechnology Co., Ltd., China), following the manufacturer's protocol. The bicistronic cassette was obtained from the pIRES vector of Clontech Laboratories, Inc. (Carlsbad, CA, USA). The p53 coding sequence was placed downstream of the internal ribosome entry site. Viral packaging and expansion were accomplished by transfection of human kidney 293 cells. Virus was purified from cell lysates according to standard protocol. Adenoviral vector encoding RPL23 (Ad-RPL23) was prepared similarly except that the coding sequence for RPL23 was directly inserted into the multicloning site of pSHUTTLE-CMV. Adenoviral vector encoding human wild-type p53 (Ad-p53) was kindly provided by Professor Shanwen Zhang and Professor Shaowen Xiao (Department of Radiotherapy, Beijing University School of Oncology, Beijing, China). The titer of the stock virus was assessed by a plaque formation assay using 293 cells. The virus solutions were stored at $-80^{\circ} \mathrm{C}$. The transduction efficiency of the adenoviral vectors in gastric cancer cells was evaluated by flow cytometry (Coulter Epics-XL; Beckman Coulter, Miami, FL, USA) using GFP-expressing adenovirus (Ad-GFP) (20). Ad-null was used as the control virus in this study.
Western blot analysis. Cells infected with adenoviral vectors at a multiplicity of infection (MOI) of 50 for $48 \mathrm{~h}$ were collected, washed twice in cold PBS, and lysed at $4^{\circ} \mathrm{C}$ in lysis buffer using protease and phosphatase inhibitors as described previously (16). Cell lysates containing $50 \mu \mathrm{g}$ total protein were resolved in $10 \%$ SDS-PAGE, transferred to PVDF membranes (Amersham Pharmacia Biotech), and probed with primary antibodies. The antibodies used included p53, MDM2, p21, PUMA and $\beta$-actin. The signals for the immunoreactive proteins were visualized on the ChemiDoc XRS system (Bio-Rad, Hercules, CA, USA) using an enhanced chemiluminescence system (Santa Cruz Biotechnology, Inc.).

Quantitative RT-PCR analysis. For quantitative real-time PCR (qRT-PCR) analysis, total RNA was extracted with TRIzol, and cDNA was prepared using the PrimerScript reverse transcriptase kit (both from Takara Bio, Inc.). Expression levels of targeted genes were analyzed by qRT-PCR using Applied Biosystems 7500 Real-Time PCR and the $S_{Y B R}{ }^{\circledR}-$ GreenER ${ }^{\mathrm{TM}}$ reagent system from Invitrogen Life Technologies as described previously. The primers used in this study are listed as follows: p53 (exon 9) forward, 5'-TATCC AGCCCTCACTCCTTC-3' and reverse, 5'-CACGGATCTGA AGGGTGAAA-3' (21); MDM2 forward, 5'-CCGGATGATC GCAGGTG-3' and reverse, 5'-AAAAGCTGAGTCAACCTG CCC-3'; p21 forward, 5'-GCGATGGAACTTCGACTTTG-3' and reverse, 5'-CAGGTCCACATGGTCTTCCT-3'; PUMA forward, 5'-GACCTCAACGCACAGTACGA-3' and reverse, 5'-CTAATTGGGCTCCATCTCG-3'; $\beta$-actin forward, 5'-GG ACTTCGAGCAAGAGATGG-3' and reverse, 5'-AGGAAGG AAGGCTGGAAGAG-3'.

Assay of cell growth and viability. Cells were plated at a density of $5 \times 10^{3}$ cells $/ 100 \mu 1$ in 96-well plates (Corning Incorporated), and were infected with adenoviral vectors at the indicated MOI $24 \mathrm{~h}$ later. Three days after adenoviral infection, cell viability was evaluated using trypan blue exclusion cell counts, and 5 days after adenoviral infection, cell growth was assessed by the 3-(4,5-dimethylthiazol-2-yl)-2,5-diphenyltetrazolium bromide (MTT) assay.

Analysis of cell apoptosis. Analysis of apoptosis was performed as described previously (16). Briefly, cells cultured in 6-well plates (Corning Incorporated) were infected with various vectors at an MOI of 50 for $48 \mathrm{~h}$, and then the cells were stained with PI/Annexin V and analyzed by flow cytometry (Coulter Epics-XL). Each analysis was performed in triplicate.

Adenovirus treatment in vivo. The protocol for the animal experiments was approved by the Institutional Animal Care and Use Committee at the Wuhan General Hospital of Guangzhou Command, People's Liberation Army and followed guidelines set forth in the National Institutes of Health Guide for the Care and Use of Laboratory Animals. MKN45 and MGC803 cells were used to establish subcutaneous xenograft tumors in BALB/c athymic nude mice (Shanghai Laboratory Animal Center of the Chinese Academy of Sciences). Briefly,

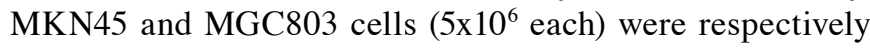
inoculated into the left and right flanks of a single nude mouse. Seven days after tumor inoculation, 24 mice bearing tumors 
with a diameter of 3-7 $\mathrm{mm}$ were randomized into 3 treatment groups: Ad-RPL23/p53, Ad-p53 and Ad-null. Mice were then treated according to the following schedule: at days 8 , 15, 22 and 29 after inoculation, each mouse was injected intratumorally with adenoviral vectors (Ad-RPL23/p53, Ad-p53 and Ad-null) at a dose of $5 \times 10^{7}$ viral particles/tumor in a volume of $50 \mu \mathrm{l}$. Serial changes in tumor volume were estimated weekly after the start of the adenovirus treatments. On day 36, after 5 measurements, animals were sacrificed by cervical dislocation under methoxyflurane anesthesia. The volume of the tumors was calculated according to the formula: Tumor volume $=\left(\right.$ length $\mathrm{x}$ width $\left.{ }^{2}\right) / 2$. Furthermore, to closely resemble the human patient, another animal model of human gastric cancer constructed orthotopically from histologically intact patient specimens was established according to a method described previously $(22,23)$. Briefly, a fresh surgical specimen derived from one patient with advanced gastric cancer was cut into pieces of $\sim 2-3 \mathrm{~mm}^{3}$ in size, and then the pieces of the tumor tissues were implanted orthotopically into the gastric wall of nude mice. Seven days after surgical orthotopic implantation, the mice were randomized into 3 groups. The grouping and treatments were performed using the same protocol as the subcutaneous xenograft model, except that the method of adenovirus administration was by intravenous injection instead of intratumoral injection. Subsequently, a long-term survival study was performed.

Statistical analysis. Quantitative results are expressed as the means \pm SD. Statistical analysis was performed using ANOVA and LSD t-test. The survival rates were estimated using the Kaplan-Meier method, and the differences were analyzed using the log-rank test to compare the resulting curves of the treatment groups. $\mathrm{P}<0.05$ was considered to indicate a statistically significant result. All statistical analyses were performed using SPSS 17.0 software (SPSS, Inc., Chicago, IL, USA).

\section{Results}

Effective infection of the adenoviral vector in gastric cancer cells. To determine the infection efficiency of the adenoviral vector in gastric cancer cells, we infected MKN45 and MGC803 cells with Ad-GFP at a MOI ranging from 10 to 200. Preliminary titration revealed a high transfer efficiency of the recombinant adenovirus in gastric cancer cells and an optimal expression at $48 \mathrm{~h}$ postinfection. At a MOI of $50,>85 \%$ gastric cells were GFP-positive without obvious adenoviral toxicity. Therefore, we performed most of our studies at $48 \mathrm{~h}$ of incubation with the optimal MOI of 50.

Ad-RPL23/p53 induces greater accumulation of p53 protein compared to Ad-p53. We initially studied the effects of ectopically expressed RPL23 on the accumulation and stability of exogenous wt p53 protein in MKN45 cells (endogenous wt p53) and MGC803 cells (endogenous mutant p53) using western blot analysis. A modest increase in wt p53 protein was noted in both the Ad-p53-transduced MKN45 and MGC803 cells, independent of the endogenous p53 status, whereas, cells treated with Ad-RPL23/p53 at the same MOI had an 4- and 3-fold higher wt p53 signal intensity than that in the Ad-p53-treated MKN45 and MGC803 cells, respectively (Fig. 1B). Since the

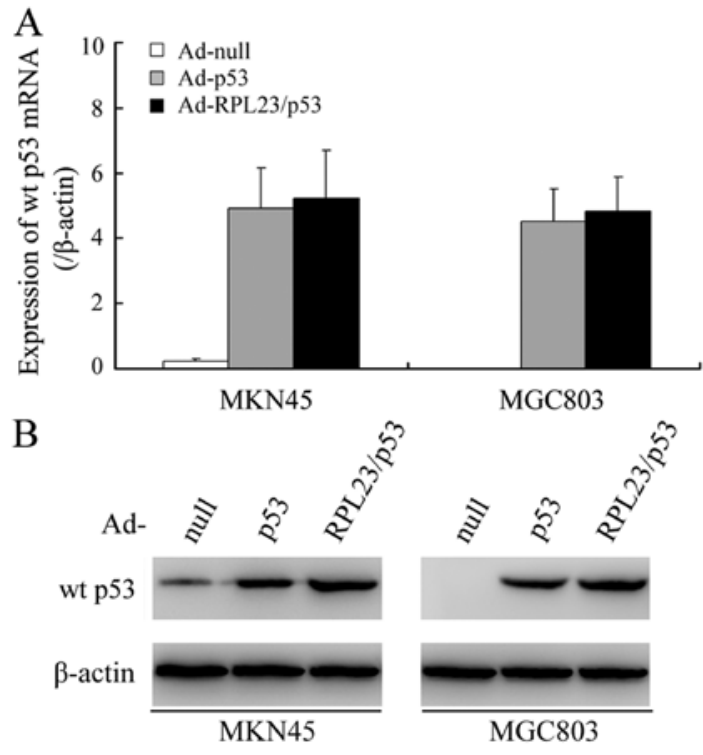

Figure 1. Expression of wt p53 mRNA and protein in gastric cancer cells (A) Quantitative RT-PCR analysis of vector-induced expression of wt p53 mRNA in MKN45 and MGC803 cells after treatment of Ad-null, Ad-p53 or Ad-RPL23/p53. The mRNA levels of wt p53 gene were normalized to the housekeeping gene $\beta$-actin using the $2^{\Delta \mathrm{Ct}}$ method $\left(\Delta \mathrm{Ct}=\mathrm{Ct}_{\beta \text {-actin }}-\mathrm{Ct}_{\mathrm{wt}}{ }_{\mathrm{p} 53}\right)$. (B) Western blot analysis of wt p53 protein expression in gastric cancer cells after the same treatments as in A. wt, wild-type.

Ad-RPL23/p53 and Ad-p53 vectors produced similar levels of p53 message when each was administered at a MOI of 50 (Fig. 1A), these results suggest involvement of post-transcriptional or post-translational control over the wt p53 protein accumulation.

Ad-RPL23/p53 is more efficient than Ad-p53 at inducing p53 target gene expression. We used qRT-PCR and western blot analysis, respectively, to analyze the mRNA and protein expression of MDM2, the cell cycle inhibitor p21, and the pro-apoptotic PUMA, all of which have p53-responsive promoters (24). Consistent with the higher levels of induced p53 protein achieved with Ad-RPL23/p53, we observed enhanced transcription of MDM2, p21 and PUMA in both MKN45 and MGC803 cells by qRT-PCR analysis after treatment with Ad-RPL23/p53 but no detectable or slight enhancement of transcription of these genes after similar treatment with Ad-p53 (Fig. 2A). As indicated by western blot analysis (Fig. 2B), levels of the MDM2, p21 and PUMA gene products also increased in both MKN45 and MGC803 cells after treatment with Ad-RPL23/p53, whereas similar Ad-p53 treatment resulted in no change or slight change in expression of these gene products.

Ad-RPL23/p53 displays more marked tumor-suppressor activity in vitro relative to Ad-p53. Next, we evaluated the in vitro tumor-suppressor activity of Ad-RPL23/p53 relative to Ad-p53 in MKN45 and MGC803 cells using MTT assay. In mutant p53 MGC803 cells, Ad-RPL23/p53 suppressed growth to a greater extent than Ad-p53, achieving complete suppression at an MOI of 100, obviously less than the required doses of Ad-p53 to achieve a similar effect (Fig. 3A). In wt p53 MKN45 cells, despite the efficient gene transfer, 


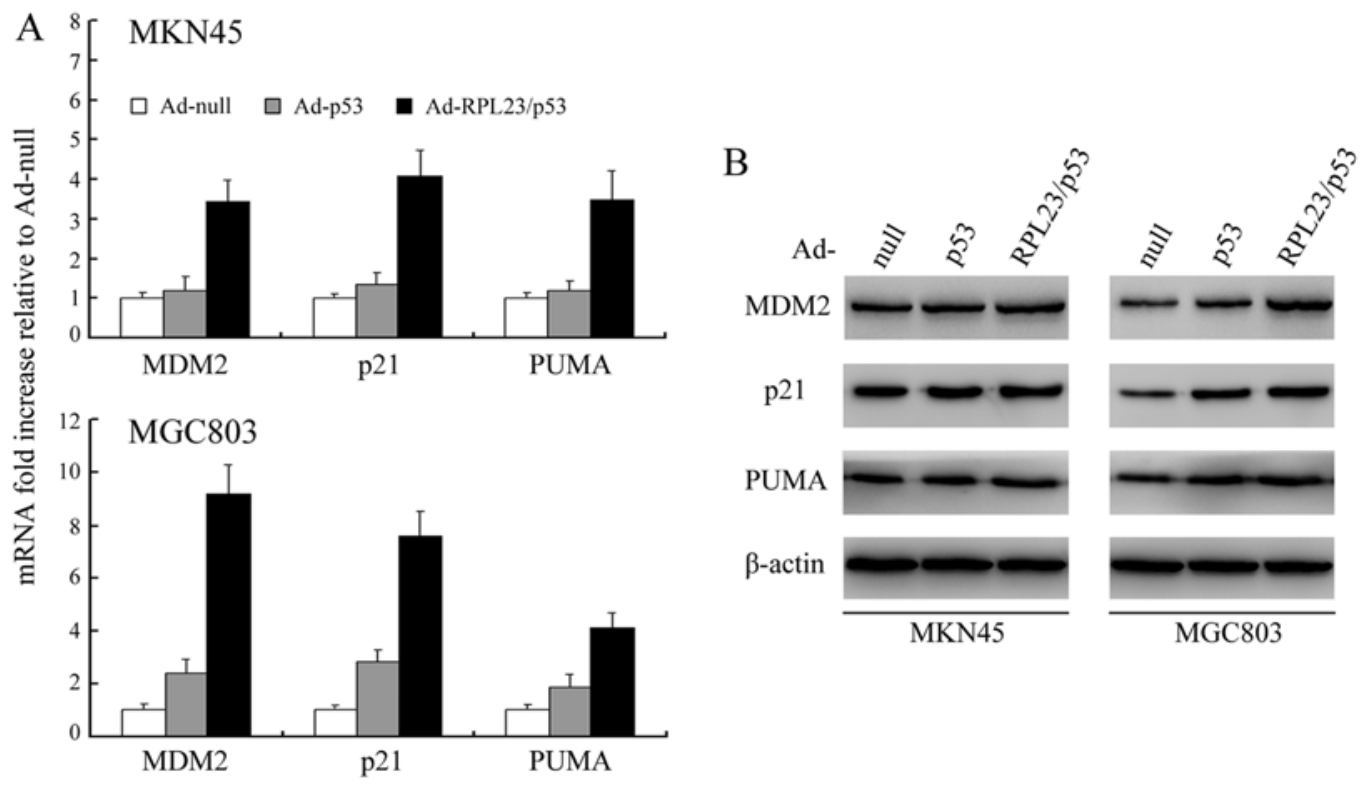

Figure 2. Quantitative RT-PCR and western blot analysis of the expression of p53 downstream target genes MDM2, p21 and PUMA in MKN45 and MGC803 cells after treatment with Ad-null, Ad-p53 or Ad-RPL23/p53. (A) The mRNA levels were measured by quantitative RT-PCR. The results are expressed as mRNA fold increase relative to that of cells treated with Ad-null. (B) Western blot analysis of the MDM2, p21 and PUMA gene products after the same treatments as in A. MDM2, murine double minute 2 .
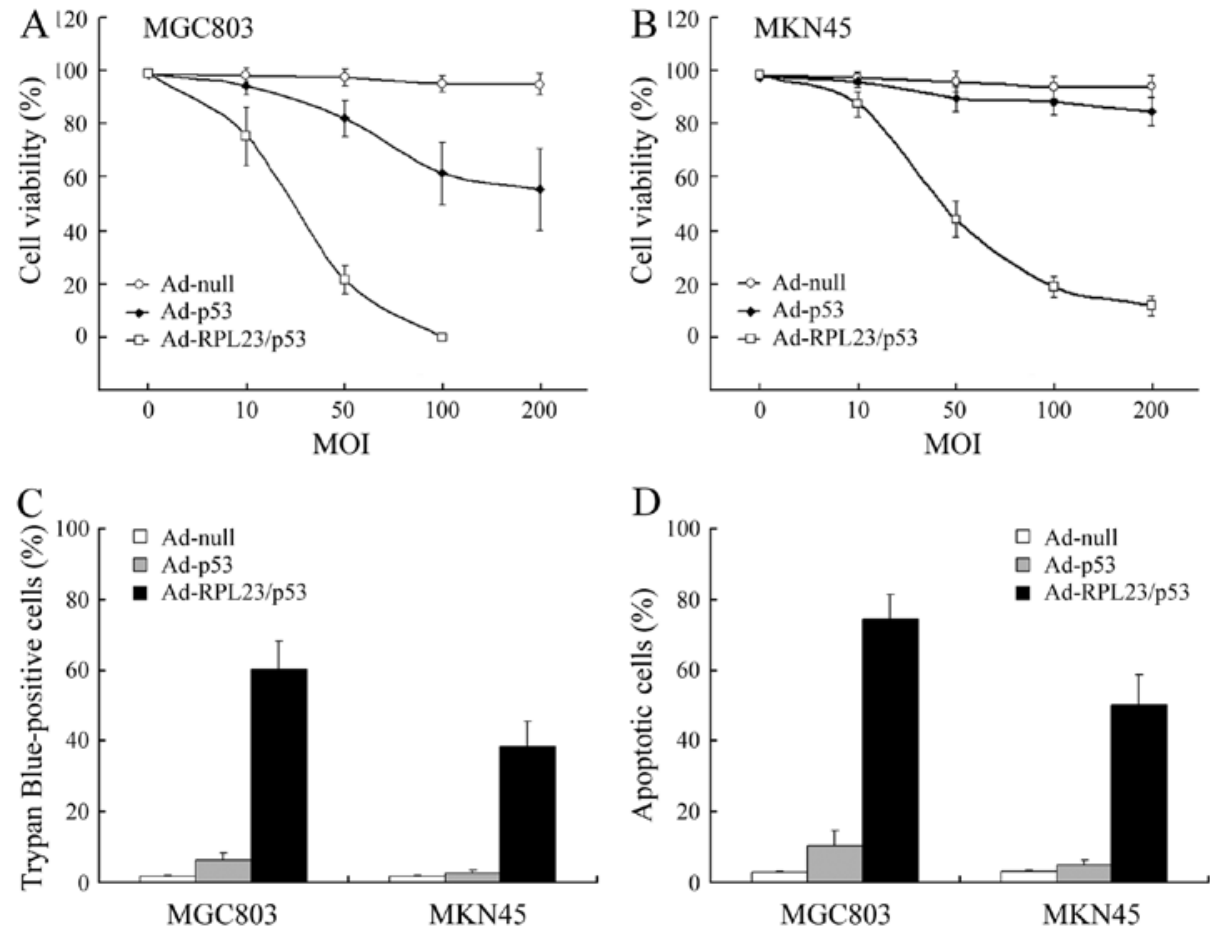

Figure 3. Analysis of cell growth and viability. (A and B) MTT assays were used to evaluate cell growth after treatment with the indicated doses of Ad-null, Ad-p53 or Ad-RPL23/p53. Data were expressed as a percentage of uninfected cells. The results are presented as means (SD) from 3 independent experiments. (C) Trypan blue exclusion assay of MKN45 and MGC803 cells after treatment with various adenoviral vectors at MOI of 50. Data points represent the average of triplicate wells. (D) FCM analysis of gastric cancer cell apoptosis after treatment as in C. Data points represent the average of triplicate wells.

the p53-specific suppression of cell growth by Ad-p53 was minimal, which has been previously reported due to the high expression of MDM2 (12). As for Ad-RPL23/p53, infection at a MOI of 50 caused a significant inhibition of cell growth $(\mathrm{P}<0.05)$ when compared with Ad-p53, which was consistent with the marked accumulation of wt p53 protein in MKN45 cells after Ad-RPL23/p53 treatment (Fig. 3B). Single ectopically expressed RPL23 protein had the capacity to induce the accumulation and stability of endogenous wt $\mathrm{p} 53$ protein; therefore, we also compared the in vitro tumor-suppressor 

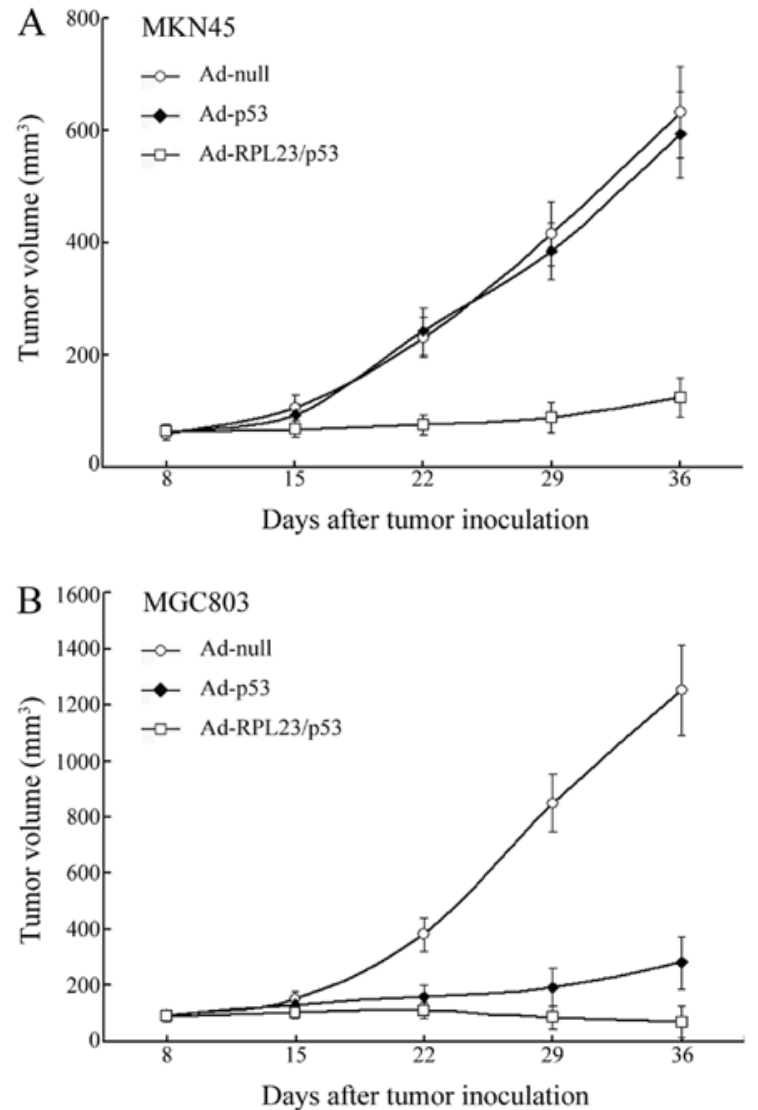

Figure 4. Suppression of tumor growth in nude mice by injection of the various adenoviruses. Tumor volume was plotted for each animal treatment group $(\mathrm{n}=8)$ (y-axis) against days after inoculation (x-axis). (A) MKN45 tumor growth. (B) MGC803 tumor growth.

activity of Ad-RPL23/p53 relative to Ad-RPL23 in wt p53 MKN45 cells. As predicated, Ad-RPL23/p53 was also more effective than Ad-RPL23 in the MKN45 cells $(\mathrm{P}<0.05$, compared with the data obtained in our previous study) (16), suggesting that the combined gene therapy strategy was more efficient at achieving tumor growth suppression.

Suppression of growth after treatment with Ad-RPL23/p53 correlates with increased cell death, as determined by trypan blue exclusion assay. Fig. 3C shows the results of the trypan blue staining carried out $72 \mathrm{~h}$ after treatment of MGC803 or MKN45 cells with Ad-null, Ad-p53 or Ad-RPL23/p53 at an MOI of 50. In the case of Ad-RPL23/p53, trypan blue-positive cells (dead cells) accounted for $\sim 60.1 \%$ (MGC803) and $\sim 38.7 \%$ (MKN45) of the cells, consistent with the marked reduction in cell viability relative to the control (Fig. 3A and B). Regarding Ad-p53, the percentage of trypan blue-positive cells remained low and accounted for $\sim 6.2 \%$ (MGC803) and 2.5\% (MKN45) of the cells, consistent with the minimal reduction in cell viability relative to the control observed after this treatment.

Apoptosis induction is firmly established as a central mechanism of p53 cancer gene therapy (25). In this study, apoptosis of gastric cancer cells treated with the various vectors at a MOI of 50 was evaluated by FCM analysis. As shown in Fig. 3D, in MGC803 and MKN45 cells, Ad-p53 treatment at a MOI of 50 had slight or little effect on apoptosis, respectively. However, at the same dose, treatment with Ad-RPL23/p53 caused more

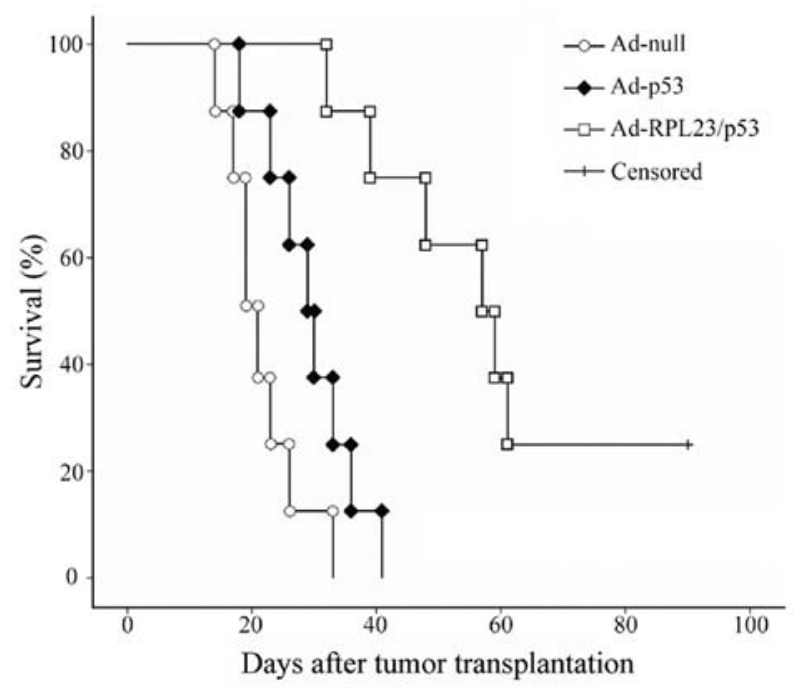

Figure 5. Kaplan-Meier survival curves for nude mice with orthotopic transplantation of fresh surgical specimens of human gastric cancer following treatment with the various adenoviral vectors.

obvious apoptosis in both cell lines $(\mathrm{P}<0.01$, compared with Ad-p53).

Ad-RPL23/p53 treatment achieves a more potent therapeutic effect than Ad-p53 in a nude mouse subcutaneous xenograft model using human gastric cancer cells. Results of the in vitro experiments led us to further investigate the in vivo effect of administration of Ad-RPL23/p53 by injection. As shown in Fig. 4A, the volumes of the MKN45 cell-derived tumors in the Ad-null and Ad-p53 groups increased 10.6- and 9.4-fold, respectively, over a period of 5 weeks, whereas in the Ad-RPL23/p53 treated mice the tumors barely progressed during that time, suggesting that the combined gene therapy displayed a significant tumor growth inhibition in vivo in the wt p53-expressing tumors which were initially resistant to gene therapy using the single p53 gene. In the case of MGC803 cell-derived tumors, treatment of Ad-p53 displayed an obvious tumor growth inhibition relative to Ad-null. However, treatment of Ad-RPL23/p53 in the same manner resulted in a more marked tumor growth inhibition relative to Ad-p53 (Fig. 4B). In the Ad-RPL23/p53 group, the MGC803 tumors began to shrink after 3 injections and disappeared in 3 of the 8 mice by the end of the experiment. The average volume of the MGC803 tumors in the Ad-RPL23/p53-treated mice (including the 3 vanishing tumors) on day 36 was $68 \pm 57.7 \mathrm{~mm}^{3}$, which was significantly less than the average tumor volume of $281 \pm 91.5 \mathrm{~mm}^{3}$ for the Ad-p53 treated mice $(\mathrm{P}<0.01)$.

Ad-RPL23/p53 treatment results in significantly longer survival than Ad-p53 treatment in an orthotopic nude mouse model constructed by using histologically intact human gastric cancer specimens. Most human gastric cancers show heterogeneous p53 expression; therefore, the animal model constructed above using established gastric cancer cell lines did not closely resemble the human patient. Therefore, we established another animal model of human gastric cancer using orthotopic transplantation of histologically intact patient specimens, and 
investigated the effect of Ad-RPL23/p53 treatment on the survival times of the tumor-bearing mice. In this study, control mice (Ad-null-treated) rapidly succumbed to the tumor burden, with a median survival of 21.5 days. Ad-p53 treatment mildly prolonged the survival of the mice, and the median survival was 29.5 days $(\mathrm{P}<0.05$, compared with Ad-null). However, as revealed in Fig. 5, relative to Ad-p53, the survival benefit was greater for the mice receiving Ad-RPL23/p53 treatment. The median survival for the 8 Ad-RPL23/p53-treated mice was 59.5 days $(\mathrm{P}<0.01$, compared with Ad-p53), and among them, 2 mice survived $>90$ days. These data suggest that the combined therapy with the RPL23 and p53 genes significantly increases patient survival even in the event of tumors with heterogeneous $p 53$ expression.

\section{Discussion}

Repair of specific genetic defects responsible for the aberrant biological behavior of cancer cells is a fascinating approach to cancer treatment. p53, due to its essential role as a tumorsuppressor, has been established as 'the gatekeeper for cell growth and division' (26). Functional integrity of the p53 gene is an essential cellular defense against neoplastic transformation. In fact, nearly all of the different types of human malignancies analyzed to date were shown to contain mutations of the p53 gene or alterations in the p53 regulating pathway (1-4). Thus, it is believed that $\mathrm{p} 53$ is an attractive target for gene replacement therapy for cancer.

Transfer of the wt p53 gene has proven effective in suppressing the proliferation of tumor cells bearing mutant p53 as noted in vitro, in animal models, and in patients with cancer. However, as many cancer cells, including gastric cancer cells, express wt p53 and most tumors are also heterogeneous with respect to their p53 status, p53 gene transfer alone may be insufficient to suppress tumor cell growth due to the general resistance of the wt p53-expressing tumor cells to p53 gene transfer. It is currently known that MDM2, the key negative regulator of $\mathrm{p} 53$, is found to be overexpressed in a variety of tumors, resulting in the inactivation of wt $\mathrm{p} 53$ protein, with an effect similar to that of mutations in the p53 gene (4). Thus, it is proposed that tumors with wt $\mathrm{p} 53$ may avoid p53-mediated enhancement of oncolysis due to a high MDM2 expression by which exogenous p53 protein is rapidly degraded. In fact, human cancer cell lines with endogenous wt p53 which are poorly responsive to the oncolysis-enhancing effect of $\mathrm{p} 53$ almost exclusively express high levels of MDM2 $(12,13,17)$. Furthermore, the MDM2 gene itself has been a downstream target of p53; it is proposed that induction of MDM2 expression by exogenous p53 may also limit the responsiveness of cancer cells to $\mathrm{p} 53$ gene transfer, in which the introduction of exogenous p53 induces the overexpression of endogenous MDM2, which, in turn, results in rapid degradation of the p53 protein.

It thus becomes clear that one way to enhance the tumorsuppression activity of p53 gene transfer is by abrogating MDM2-mediated p53 inactivation. Nutlin, the first identified small-molecule MDM2 antagonists that inhibits MDM2-p53 interaction, has been demonstrated to enhance tumor cell killing following adenoviral-mediated p53 gene transfer $(27,28)$. Combined delivery of one addition gene with a product that could inhibit the MDM2-p53 feedback loop is another flexible strategy. Because of its well-characterized ability to inhibit MDM2-mediated p53 degradation, Tango et al (29) and Huang et al (30) hypothesized that co-transduction of p14 ${ }^{\mathrm{ARF}}$ may enhance the tumor-suppressive activity of p53 gene transfer by increasing $\mathrm{p} 53$ protein stability. By using in vitro and in vivo approaches, they demonstrated that the therapeutic efficacy of the combined transduction of p53 with p14 ${ }^{\mathrm{ARF}}$ was superior to transduction with p53 alone.

Since the RPL23 protein has a similar function with p14 ${ }^{\mathrm{ARF}}(14,15)$, we hypothesized that co-transduction of RPL23 could also enhance the therapeutic efficacy of the adenoviral-mediated $\mathrm{p} 53$ gene transfer through protecting exogenous wt $\mathrm{p} 53$ from MDM2-mediated inactivation. Using an in vitro system with cultured cells, we observed that gastric cancer MKN45 and MGC803 cells, regardless of the p53 status, showed marked growth arrest and apoptosis after treatment with a bicistronic adenovirus expressing both the RPL23 and p53 genes (Ad-RPL23/p53), while, at the same infectious dose, the efficacies of the single gene vector for $\mathrm{p} 53$ (Ad-p53) were not detectable or minimal. Ad-RPL23/p53 and Ad-p53 induced similar levels of p53 message under similar treatment conditions, while, consistent with the function of ectopic RPL23 protein in stabilizing p53, the accumulation of p53 protein in cells treated with Ad-RPL23/p53 was more significant than that achieved by a similar treatment with Ad-p53. Accompanied with this, increased RNA and protein expression of the p53 downstream target genes MDM2, p21 and PUMA was observed. The in vivo data further indicated that co-expression of RPL23 and p53 may have greater antitumor activity than p53 alone. In a subcutaneous xenograft model established from MKN45 (endogenous wt p53) and MGC803 cells (endogenous mutant p53), we found that Ad-RPL23/p53 treatment achieved a more potent therapeutic effect relative to Ad-p53. To better estimate the usefulness of Ad-RPL23/p53 for patients with gastric cancer which usually exhibit heterogeneous p53 expression, we established another animal model of human gastric cancer using orthotopic transplantation of histologically intact patient specimens. Ad-RPL23/p53 treatment led to prolonged survival when compared to that obtained by Ad-p53. Since the orthotopic transplantation model showed various manifestations similar to the tumor behavior in patients, including metastasis, the prolonged survival after Ad-RPL23/p53 treatment indicated that the combined gene therapy suppressed not only tumor growth but also metastasis.

In conclusion, the present study suggests that combination gene therapy with adenoviral vector-mediated RPL23 and p53 is feasible and more effective than p53 alone. More importantly, therapeutic efficacy of the bicistronic adenovirus can be achieved in both p53 mutant cancer cells but also in p53 wild-type cancer cells. Therefore, this strategy has broad application for cancer treatment by ensuring the induction of p53 protein accumulation in malignant cells regardless of the p53 genotypes.

\section{Acknowledgements}

The present study was supported by the National Foundation of Natural Sciences, China (no. 81101533) and the China 
Postdoctoral Science Foundation (nos. 201104755 and 20100481468).

\section{References}

1. Cheok CF, Verma CS, Baselga J and Lane DP: Translating p53 into the clinic. Nat Rev Clin Oncol 8: 25-37, 2011.

2. Brown CJ, Lain S, Verma CS, Fersht AR and Lane DP: Awakening guardian angels: drugging the p53 pathway. Nat Rev Cancer 9: 862-873, 2009.

3. Lane DP and Lain S: Therapeutic exploitation of the p53 pathway. Trends Mol Med 8 (Suppl 4): S38-S42, 2002.

4. Lane DP, Cheok CF and Lain S: p53-based cancer therapy. Cold Spring Harb Perspect Biol 2: a001222, 2010.

5. Momand J, Zambetti GP, Olson DC, George D and Levine AJ: The mdm-2 oncogene product forms a complex with the p53 protein and inhibits p53-mediated transactivation. Cell 69: 1237-1245, 1992.

6. Haupt Y, Maya R, Kazaz A and Oren M: Mdm2 promotes the rapid degradation of p53. Nature 387: 296-299, 1997.

7. Kubbutat MH, Jones SN and Vousden KH: Regulation of p53 stability by Mdm2. Nature 387: 299-303, 1997.

8. Marine JC and Lozano G: Mdm2-mediated ubiquitylation: p53 and beyond. Cell Death Differ 17: 93-102, 2010.

9. Moll UM and Petrenko O: The MDM2-p53 interaction. Mol Cancer Res 1: 1001-1008, 2003.

10. Shangary S and Wang S: Targeting the MDM2-p53 interaction for cancer therapy. Clin Cancer Res 14: 5318-5324, 2008.

11. Wiman KG: Strategies for therapeutic targeting of the p53 pathway in cancer. Cell Death Differ 13: 921-926, 2006.

12. van Beusechem VW, van den Doel PB and Gerritsen WR: Conditionally replicative adenovirus expressing degradationresistant p53 for enhanced oncolysis of human cancer cells overexpressing murine double minute 2. Mol Cancer Ther 4: 1013-1018, 2005.

13. Nishizaki M, Sasaki J, Fang B, Atkinson EN, Minna JD, Roth JA and Ji L: Synergistic tumor suppression by coexpression of FHIT and p53 coincides with FHIT-mediated MDM2 inactivation and p53 stabilization in human non-small cell lung cancer cells. Cancer Res 64: 5745-5752, 2004

14. Dai MS, Zeng SX, Jin Y, Sun XX, David L and Lu H: Ribosomal protein L23 activates p53 by inhibiting MDM2 function in response to ribosomal perturbation but not to translation inhibition. Mol Cell Biol 24: 7654-7668, 2004.

15. Jin A, Itahana K, O'Keefe K and Zhang Y: Inhibition of HDM2 and activation of p53 by ribosomal protein L23. Mol Cell Biol 24: 7669-7680, 2004.

16. Zhang Y, Shi Y, Li X, et al: Inhibition of the p53-MDM2 interaction by adenovirus delivery of ribosomal protein L23 stabilizes p53 and induces cell cycle arrest and apoptosis in gastric cancer. J Gene Med 12: 147-156, 2010.
17. Ohashi M, Kanai F, Ueno H, et al: Adenovirus mediated p53 tumour suppressor gene therapy for human gastric cancer cells in vitro and in vivo. Gut 44: 366-371, 1999.

18. Jiang $\mathrm{XH}$, Wong BC, Lin MC, et al: Functional p53 is required for triptolide-induced apoptosis and AP-1 and nuclear factor- $\kappa \mathrm{B}$ activation in gastric cancer cells. Oncogene 20: 8009-8018, 2001.

19. Sun S,Li XM,Li XD and Yang WS: Studies on inducing apoptosis effects and mechanism of CIK cells for MGC-803 gastric cancer cell lines. Cancer Biother Radiopharm 20: 173-180, 2005.

20. Kim HJ, Cho HI, Han YH, et al: Efficient transduction with recombinant adenovirus in EBV-transformed B lymphoblastoid cell lines. J Biochem Mol Biol 37: 376-382, 2004.

21. Yang HY, Zhang WG, Ma LP, Wang SW and Zhang ZY: An approach to enhancing the phototoxicity of a novel hypocrellin congener to MGC803 cells. Dyes Pigments 51: 103-110, 2001.

22. Furukawa T, Kubota T, Watanabe M, Kitajima $M$ and Hoffman RM: Orthotopic transplantation of histologically intact clinical specimens of stomach cancer to nude mice: correlation of metastatic sites in mouse and individual patient donors. Int J Cancer 53: 608-612, 1993.

23. Shi J, Wei PK, Zhang S, et al: OB glue paste technique for establishing nude mouse human gastric cancer orthotopic transplantation models. World J Gastroenterol 14: 48004804, 2008.

24. Ozaki T and Nakagawara A: p53: the attractive tumor suppressor in the cancer research field. J Biomed Biotechnol 2011: 603925, 2011.

25. Amaral JD, Xavier JM, Steer CJ and Rodrigues CM: Targeting the p53 pathway of apoptosis. Curr Pharm Des 16: 2493-2503, 2010.

26. Levine AJ: p53, the cellular gatekeeper for growth and division. Cell 88: 323-331, 1997.

27. Tisato V, Norcio A, Voltan R, Celeghini C, Zella D and Secchiero P: MDM2 non-genotoxic inhibitors as innovative therapeutic approaches for the treatment of pediatric malignancies. Curr Med Chem 20: 2226-2236, 2013.

28. Graat HC, Carette JE, Schagen FH, et al: Enhanced tumor cell kill by combined treatment with a small-molecule antagonist of mouse double minute 2 and adenoviruses encoding p53. Mol Cancer Ther 6: 1552-1561, 2007.

29. Tango Y, Fujiwara T, Itoshima T, et al: Adenovirus-mediated p14 ${ }^{\mathrm{ARF}}$ gene transfer cooperates with Ad5CMV-p53 to induce apoptosis in human cancer cells. Hum Gene Ther 13: 1373-1382, 2002.

30. Huang Y, Tyler T, Saadatmandi N, Borgstrom P and Gjerset RA: Enhanced tumor suppression by a p14ARF/p53 bicistronic adenovirus through increased p53 protein translation and stability. Cancer Res 63: 3646-3653, 2003. 